\title{
Frecuencia y distribución de maloclusión en pa- cientes adultos jóvenes del Hospital Odontológico de la Facultad de Odontología de la UNNE.
}

Frequency and distribution of malocclusion in young adult patients of hospital dentistry faculty of Dentistry UNNE.
Frequência má oclusão e distribuição de jovens adultos em hospital do paciente dental faculdade de Odontologia UNNE.
Fecha de Recepción

13 de septiembre de 2013

Alejandro Javier Ponce

Becario de Investigación. Consejo Interuniversitario Nacional. Cátedra Metodología de la Investigación. UNNE.

Alina Noelia Peláez Auxiliar Docente. Dedicación Exclusiva. Cátedra Metodología de la Investigación. UNNE. E-mail:alin_pelaez@yahoo.com.ar; anpelaez@odn.unne.edu.ar.

Silvia Matilde Mazza Profesora Titular. Cátedra Metodología de la Investigación. UNNE. E-mail:smmazza@unne.edu.ar

Lugar de Trabajo Facultad de Odontología. Universidad Nacional del Nordeste. Av. Libertad 5450. CP 3400. Corrientes. Argentina.
Aceptado para su publicación

22 de octubre de 2013
El objetivo de este estudio fue determinar la frecuencia de los diferentes tipos de maloclusión aplicando la clasificación de Angle y su distribución según sexo, en pacientes adultos jóvenes del Hospital Odontológico de la Facultad de Odontología de la UNNE. Se realizó un estudio observacional, descriptivo de corte transversal, en 53 pacientes, entre 16 y 35 años, que concurrieron al Hospital Odontológico de la Facultad de Odontología de la UNNE. Se determinó la relación molar según clasificación de Angle. Para el análisis estadístico se emplearon técnicas gráficas de análisis descriptivo de datos y prueba de chi cuadrado. La clase I se presentó con mayor frecuencia en ambos sexos, con un $32,08 \%$ en el femenino y un $24,53 \%$ en el masculino, la clase II en el sexo femenino fue de $15,09 \%$ y $9,43 \%$ en el masculino, por último se presentó la clase III, con un $11,32 \%$ en el sexo masculino y $7,55 \%$ en el femenino. Se realizó la prueba de chi cuadrado (grados de libertad $=2 ; p=0,99$ ) determinando que no existe asociación entre frecuencia de maloclusión y sexo. Conclusión: La maloclusión más frecuente fue clase I, seguida de clase II y por último clase III.

\section{Palabras claves}

Anomalías de la oclusión, relación molar, clasificación de Angle. 
REVISTA FACULTAD DE ODONTOLOGÍA

ISSN No 1668-7280 - Vol. VI No 2 - 2013

23
INVESTIGACIÓN

Frecuencia y distribución de maloclusión en pacientes adultos jóvenes del Hospital Odontológico de la Facultad de Odontología de la UNNE

\section{Summary}

The aim of this study was to determine the frequency of different types of malocclusion Angle's classification applied and their distribution by sex in young adult patients of Dental Hospital, Faculty of Dentistry UNNE. We performed an observational, descriptive, cross-sectional, in 53 patients, between 16 and 35 years, who attended the Hospital Dentistry, Faculty of Dentistry UNNE. Molar ratio was determined according to Angle classification. For statistical analysis, graphical techniques were used descriptive data analysis and chi square test. The class I was presented with greater frequency in both sexes, with $32.08 \%$ among females and $24.53 \%$ in males, class II in females was $15.09 \%$ and $9.43 \%$ in the male finally presented the class III with a $11.32 \%$ in males and $7.55 \%$ in females. We performed chi-square test ( $\mathrm{df}=2, \mathrm{p}=0.99$ ) determining that there is no association between frequency of malocclusion and sex. Conclusion: The most frequent malocclusion class I, class II followed and finally class III.

\section{Keywords}

Anomalies of occlusion, molar relation, Angle classification.

\section{Resumo}

O objetivo deste estudo foi determinar a freqüência de diferentes tipos de classificação da má oclusão de Angle aplicada e sua distribuição por sexo em pacientes jovens e adultos do Hospital Odontologia da Faculdade de Odontologia UNNE. Foi realizado um estudo observacional, descritivo, transversal, em 53 pacientes, entre 16 e 35 anos, que participou do Hospital Odontologia da Faculdade de Odontologia UNNE. Razão molar foi determinado de acordo com a classificação de Angle. Para a análise estatística, técnicas gráficas foram utilizadas análise descritiva dos dados e teste do qui-quadrado. A classe I foi apresentado com maior freqüência em ambos os sexos, com $32,08 \%$ no sexo feminino e $24,53 \%$ do sexo masculino, classe II nas fêmeas foi de $15,09 \%$ e $9,43 \%$ em o macho finalmente apresentado a classe III, com uma II,32\% nos machos e 7,55\% nas fêmeas. Foi realizado o teste do qui-quadrado $(g l=2, p=0,99)$ determinar que não existe uma associação entre a freqüência de má oclusão e sexo. Conclusão: A classe de má oclusão mais freqüente I, classe II seguido e, finalmente, classe III.

\section{Palavras chave}

Anormalidades de oclusão, uma razão molar, classificação de Angle.

\section{Introducción}

La maloclusión se define como una oclusión anormal, en la cual los dientes no están en una posición adecuada en relación con los dientes adyacentes del mismo maxilar, o entre dientes opuestos con los maxilares en cierre. No es una enfermedad sino una variación morfológica, la cual puede o no estar asociada a una condición patológica'. Según la Organización Mundial de la Salud (OMS), ocupan el tercer lugar mundial en problemas de salud bucal'2. La mayoría de las alteraciones bucales y en particular, las maloclusiones no son de riesgo de vida, pero por su prevalencia e incidencia, son consideradas un problema de salud pública ${ }^{3,4}$ sin embargo, su importancia se establece no sólo por el número de personas que la presentan, sino además, por los efectos nocivos que pueden generar en la cavidad oral ${ }^{5,6}$. Los datos epidemiológicos confirman este problema, ya que estadísticamente, se presentan con tasas de prevalencia considerablemente altas, con más del $60 \%$ de la población a nivel mundial afectada por anomalías oclusales ${ }^{7}$. Es por ello, que la clasificación de maloclusión es una herramienta elemental en el diagnóstico, llevando a determinar las necesidades de tratamiento y condiciones propias de cada individuo $0^{8,9}$.

La primera clasificación fue presentada por Edward Angle en 1899, la cual es importante hasta nuestros días, ya que es sencilla, práctica y ofrece una visión inmediata del tipo de anomalía oclusal a la que se refiere. Esta clasificación se basa en la hipótesis de que el primer molar es la pieza dentaria más estable de la dentición ${ }^{10}$ se fundamenta en la relación anteroposterior de las arcadas dentarias, considerando, la relación entre el primer molar permanente superior y el primer molar permanente inferior. Este método, contempla tres categorías: la maloclusión clase I, 
II y IIII, I2.

Se han propuesto un gran número de clasificaciones, pero a pesar de sus méritos ninguna ha reemplazado al sistema de Angle, actualmente sigue siendo el más utilizado, empleado por numerosos estudios epidemiológicos en la población mundial, determinando la prevalencia según relación oclusal, ya que existe una gran variabilidad en la manera como se distribuye de acuerdo a la edad y $\operatorname{sexo}^{13}$. Es necesario caracterizar a las anomalías oclusales más prevalentes según su tipo, para dedicar a ellas los recursos y esfuerzos educativos haciendo énfasis en aquellas situaciones clínicas frecuentes que sean susceptibles de ser prevenidas, otorgando trascendencia a este nivel de atención. Igualmente, es importante el diagnóstico de maloclusión en adultos jóvenes, para definir prioridades y pautas de tratamiento temprano, aportando una valiosa información que permita establecer prioridades en el acceso a los recursos asistenciales.

El objetivo de este estudio fue determinar la frecuencia de los diferentes tipos de maloclusión aplicando la clasificación de Angle y su distribución según sexo, en pacientes adultos jóvenes del Hospital Odontológico de la Facultad de Odontología de la UNNE.

\section{Materiales y Métodos}

Se diseñó un estudio observacional, descriptivo de corte transversal. El protocolo de investigación fue aprobado por el Comité de Bioética de la Facultad de Odontología de la UNNE.

La muestra se constituyó con la selección de 53 pacientes adultos jóvenes que concurrieron al Hospital Odontológico "Santa Apolonia" de la Facultad de Odontología de la UNNE, en edades comprendidas entre 16 y 35 años, de acuerdo a la clasificación de los métodos básicos de la OMS, ${ }^{14}$ elegidos mediante un muestreo oportunístico, que se ajustaron a criterios de inclusión/exclusión, que se detallan.

Criterios de Inclusión: Pacientes con maloclusión clasificable por Angle ${ }^{12}$, aquellos que brindaron su consentimiento para la utilización de los datos de exploración y participación del estudio (Padres o tutores en caso de pacientes menores). Criterios de Exclusión: Pacientes con dentición temporaria o mixta, padecer alguna patología sis- témica significativa, haber recibido en el pasado, o estar recibiendo cualquier tipo de tratamiento ortopédico u ortodóncico.

En el examen clínico, se determinó el tipo de maloclusión según relación molar en clase I, II y III, siguiendo el criterio de clasificación de Angle ${ }^{12}$, todos los registros clínicos fueron realizados por un único examinador, calibrado y entrenado a tal efecto.

Considerando Clase I de Angle: En presencia de una relación anteroposterior normal de los primeros molares permanentes, cuando la cúspide mesiovestibular del primer molar superior se encontró en la misma línea que el surco mesiovestibular del primer molar inferior (Normoclusión).

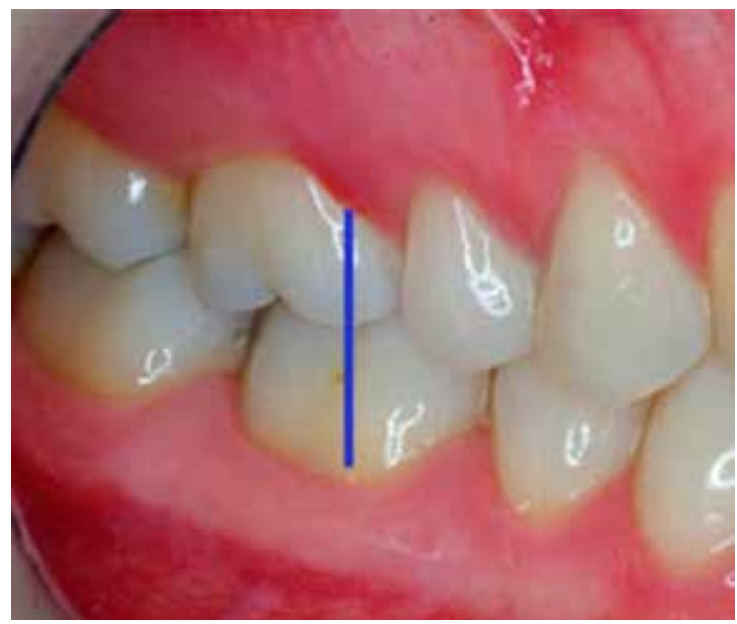

Figura I. Relación Molar Clase I de Angle.

Clase II de Angle: Ante la presencia de una relación distal de los primeros molares inferiores, cuando el surco mesiovestibular del primer molar inferior se encontró detrás de la cúspide mesiovestibular del primer molar superior (Distoclusión). (Figura $\mathrm{N}^{\circ} 2$ )

Clase III de Angle: Se identificó por la presencia de una relación mesial de los primeros molares inferiores, donde el surco mesiovestibular del primer molar inferior se encontró por delante de la cúspide mesiovestibular del primer molar superior (Mesioclusión). (Figura $\mathrm{N}^{\circ} 3$ )

Una vez determinado el tipo de maloclusión según la clasificación de Angle ${ }^{12}$ los pacientes fueron distribuidos por sexo. Para el análisis de los datos se emplearon técnicas gráficas de análisis descriptivo de datos y prueba de chi cuadrado, mediante el software estadístico Infostat Versión 


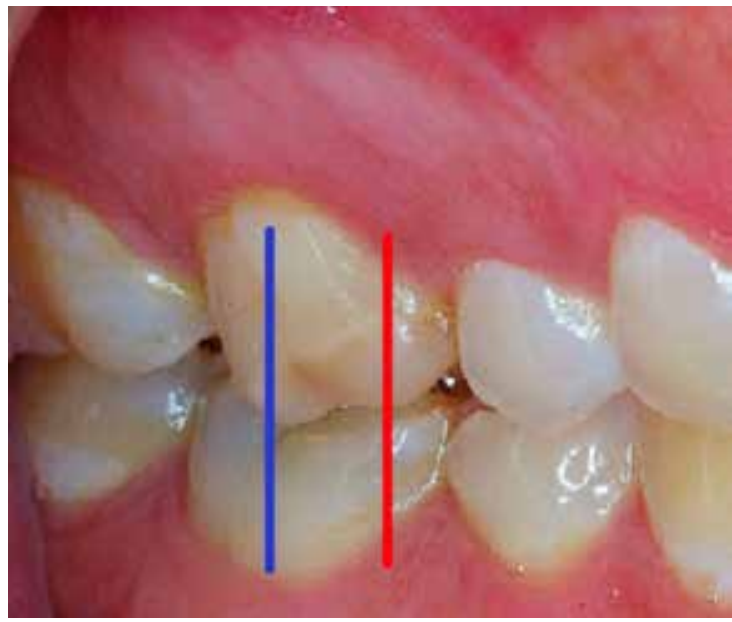

Figura 2. Relación Molar Clase II de Angle.

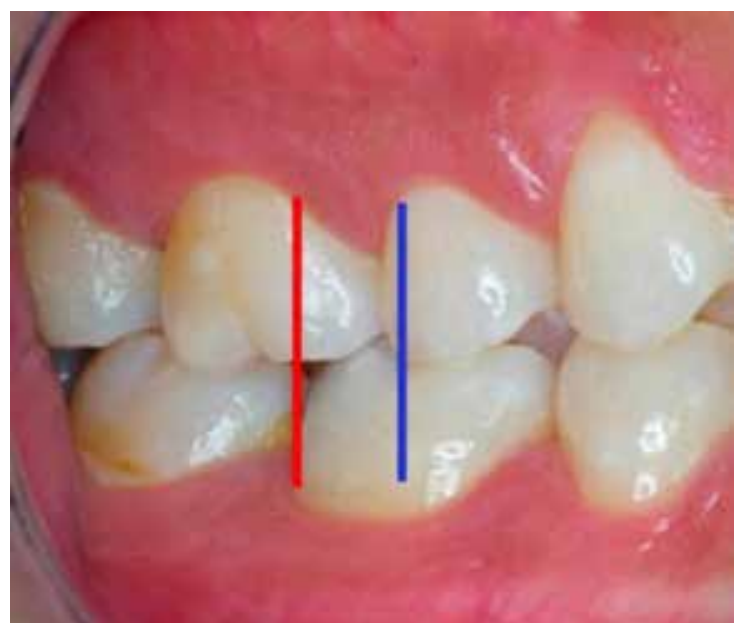

Figura 3. Relación Molar Clase III de Angle.

$2011^{15}$

\section{Resultados}

Fueron evaluados un total de 53 pacientes adultos jóvenes en edades comprendidas entre 16 y 35 años, con un promedio de 23,3 años de edad, donde el $57 \%$ presentó maloclusión clase I, seguida de clase II con un $24 \%$ y clase III con el 19\%. (Figura $N^{\circ} 4$ )

En la distribución por sexo, se pudo observar que 29 (55\%) correspondieron al sexo femenino y 24 (45\%) al masculino. La clase I se presentó con mayor frecuencia en ambos sexos, con un $32,08 \%$ en el femenino y un $24,53 \%$ en el masculino, la clase II en el sexo femenino fue de $15,09 \%$ y $9,43 \%$ en el masculino, por último se presentó

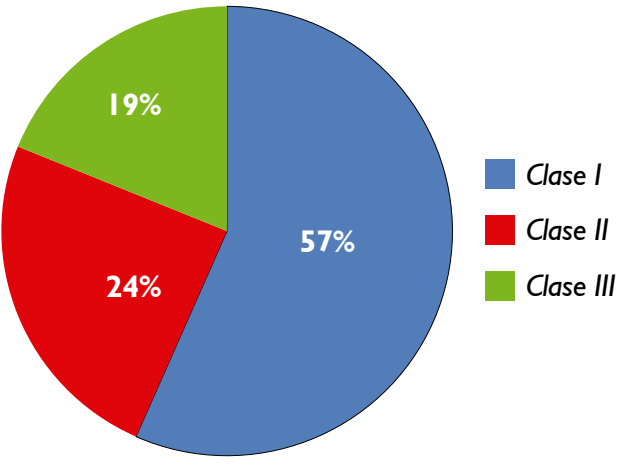

Figura 4. Prevalencia de Maloclusión Según Clasificación de Angle en adultos jóvenes del Hospital Odontológico de la Facultad de Odontología de la UNNE.

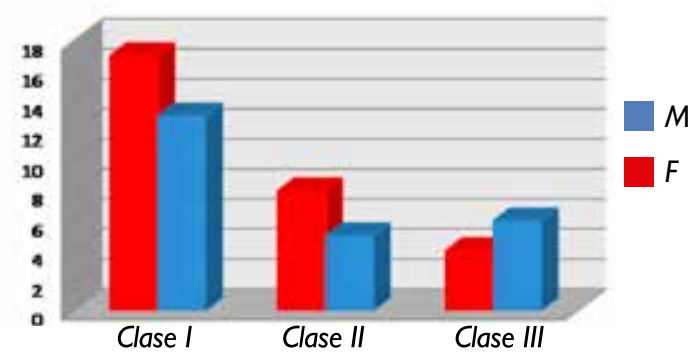

Figura 5. Prevalencia de Maloclusión Según Clasificación de Angle en adultos jóvenes del Hospital Odontológico de la Facultad de Odontología de la UNNE.

la clase III, con un I I,32\% en el sexo masculino y $7,55 \%$ en el femenino. (Figura $N^{\circ} 5$ )

Los valores obtenidos fueron analizados a través de la prueba de chi cuadrado ( $\mathrm{g}$. I. = 2; $\mathrm{P}$ $=0,99$ ) determinando que no existe asociación entre frecuencia de maloclusión y sexo.

\section{Discusión}

La clasificación de maloclusión según Angle ${ }^{12}$ sigue siendo la más utilizada a nivel mundial, a pesar de sus limitaciones para describir las características particulares de las anomalías de la oclusión en cada paciente, pero su valor radica en la descripción de la morfología general de la maloclusión, que orienta su diagnóstico y enfoque terapéutico, presentando como ventaja su sencillez y alta reproducibilidad, siendo parámetro para múltiples estudios epidemiológicos.

En este estudio, la maloclusión más prevalente según la clasificación de Angle en adultos jóvenes fue la clase I con un $57 \%$, seguida 
de clase II con $24 \%$ y la menos prevalente fue la clase III con el 19\%. Estos datos coinciden con los registros encontrado por Ayala Pérez, ${ }^{16}$ en un estudio realizado en pacientes adolescentes del Servicio Odontológico de la Clínica Manuel Angulo de Holguín, donde la clase I fue la más frecuente representada por un $50 \%$, seguida de la clase II con $40,5 \%$ y por último la clase III con $9,5 \%$. Diversos autores concuerdan que la clase I es la que se presenta con mayor frecuencia, lo cual se ajusta con los resultados obtenidos en este estudio.

Folaranmi $\mathrm{N}$ y Okeke $\mathrm{A},{ }^{17}$ encontraron una prevalencia del $60 \%$ de clase I en 75 pacientes adultos jóvenes, que asistieron a la Unidad de Ortodoncia del Hospital de la Universidad de Enseñanza de Nigeria. También Gelgör IE, ${ }^{18}$ evaluó la maloclusión en una población de adolescentes de Turquía, donde el $45 \%$ tenía clase I, el $44,7 \%$ clase II y el $10.3 \%$ clase III. Así como Martins MG, ${ }^{19}$ que clasificó la maloclusión en 274 alumnos de 10 a 12 años en una escuela de la ciudad de Fortaleza Brasil, donde halló una elevada prevalencia del $76,5 \%$ de clase I, el $22.3 \%$ clase II y el $4.2 \%$ clase III. Similares resultados presentó un estudio realizado en un grupo de adolescentes Nigerianos donde manifestaron un $74,5 \%$ clase I, un $13,7 \%$ clase II y un II,8\% clase $I \mathrm{II}^{20}$. Estos valores concuerdan con los de Montiel, ${ }^{21}$ el cual evaluó la prevalencia de maloclusión según Angle, pero en una población de niños mexicanos en edades entre 6 a 12 años, presentando un $68 \%$ de clase I, un $32 \%$ de clase II y un $9 \%$ clase III.

A diferencia de Pantaleão dos Santos MR, ${ }^{22}$ el cual demostró una elevada prevalencia de maloclusión clase II, evaluando las condiciones de oclusión en 170 escolares de 12 años, examinados por el Proyecto Salud Bucal Brasil, donde el 73,68\% presentó clase II de Angle, un 21,18\% clase I y solo un 5, I $4 \%$ clase III.

En cuanto a la distribución por sexo, pudimos observar que en el sexo femenino prevaleció la clase I, seguida de la clase II y por último clase III, mientras que en el sexo masculino la clase I se presentó con mayor frecuencia, luego la clase III y por último la clase II. Roa Gonzales, ${ }^{23}$ realizó en Monterrey México, un estudio descriptivo en $28 \mathrm{I}$ adolescentes de prevalencia y distribución de maloclusión por sexo según la clasificación de Angle, determinando que en el sexo masculino la maloclusión de mayor prevalencia fue la clase I con un porcentaje de $42,6 \%$, seguida de la clase III en un $35,3 \%$ y la clase II con un $22,1 \%$, siendo estos datos aproximados a los resultados obtenidos en este trabajo referente al sexo masculino, en cambio en el sexo femenino determinó que la clase I se manifestó en un $44,8 \%$, seguida de la clase III en un $35,9 \%$ y la clase II en $19,3 \%$, muy diferentes a los hallados en este estudio ya que en el sexo femenino la clase I con $32,08 \%$ fue las más frecuente, seguida de la clase II con un 15,09\% y por último la clase III con un bajo porcentaje de $7,55 \%$ en comparación a un $35,9 \%$ hallado por Roa Gonzáles ${ }^{23}$ en el sexo femenino.

\section{Conclusión}

- La maloclusión clase I fue la más prevalente.

- En el sexo femenino fue más frecuente la clase I, seguida de la clase II y por último la clase III.

- En el sexo masculino prevaleció la clase I seguida de la clase III y por último la clase II. 
INVESTIGACION

Frecuencia y distribución de maloclusión en pacientes adultos jóvenes del Hospital Odontológico de la Facultad de Odontología de la UNNE

\section{Bibliografía}

I. Mafla A. C., Barrera D. A., Muñoz G. M. Maloclusión y necesidad de tratamiento ortodóntico en adolescentes de Pasto, Colombia. Rev. Fac. Odontol Univ. Antioq 20I I; 22 (2): I73-I85.

2. Álvarez R; Bolasco A; Buño AG; Lúgaro A; Pascuali L; Santos M. Son las maloclusiones un problema de salud pública en el Uruguay. Acta odontol 2010; 7 (I): 57-68.

3. Del Castillo AA; Mattos Vela MA; Del Castillo RA; Del Castillo Mendoza C. Maloclusiones en Niños y Adolescentes de Caseríos y Comunidades Nativas de la Amazonía de Ucayali, Perú. Rev Peru Med Exp Salud Pública. 20I I; 28 (I): 87-9l.

4. Shivakumar KM; Chandu GN; Subba Reddy VV; Shafiulla MD. Prevalence of malocclusion and orthodontic treatment needs among middle and high school children of Davangere city, India by using Dental Aesthetic Index. J Indian Soc Pedod Prev Dent. 2009; 27 (4): 2II-8.

5. Bermúdez P; Arbeláez AM; Pineda Guerra JF; Correa DM; Acosta Fernández ML; y col. Perfil Epidemiológico de la Oclusión Dental, en Escolares de 6 a 12 años, del Colegio Universidad Cooperativa de Colombia, Corregimiento de San Antonio de Prado. Rev. Colombiana de Investigación en Odontología 20II; 2 (5): 68-73.

6. García García VJ, Ustrell Torrent JM, Sentís Vilalta J. Evaluación de la maloclusión, alteraciones funcionales y hábitos orales en una población escolar: Tarragona y Barcelona. Av. Odontoestomatol 20I I; 27 (2): 75-84.

7. Cano C; Rosas C; Gutiérrez N; Velásquez Y; Godoy S; Quirós $O$ y col. Frecuencia de maloclusión en niños de 5 a 9 años en una zona rural del Estado Guárico período 2007-2008. Revista Latinoamericana de Ortodoncia y Odontopediatria "Ortodoncia.ws edición electrónica junio 2008. Obtenible en: www.ortodoncia.ws. Consultada, 23/03/201I.

8. Elizondo Dueñaz R; Yudovich Burak M; Aguilar Saavedra M; Meléndez Ocampo A. Propuesta de un índice de maloclusiones Invalidantes con Potencial de Deterioro y su Aplicación. Rev. Odont. Mexicana. 201 I; 15 (2): 77-95.

9. Rabie ABM; Cert Ortho BDS; Yan Gu BDS. Diagnostic criteria for pseudo-Class III maloclusión. American Journal of Orthodontics \& Dentofacial Orthopedics. 2000; 45 (I): I-9.

10. Miguel-Neto AB; Nishio C; Mucha JN. Agreement evaluation of a newly proposed system for malocclusion classification. Int. J. Odontostomat. 2010; 4 (I): 33-4I.
II. Cano Rosás M. Libro de Prácticas Ortodoncia I. Primera Edición. Ed Universidad Salamanca. España. 2010; pp 48-54.

12. Ugalde Morales FJ. Clasificación de la maloclusión en los planos anteroposterior, vertical y transversal. Rev ADM. 2007; 64 (3): 97-109.

13. Murrieta Pineda JF, Cruz Diaz Pa, López Aguilar J, Marsues dos santos MJ, Zurita Murillo V. Prevalencia de maloclusiones dentales en un grupo de adolescentes mexicanos y su relación con edad y género. Acta Odont Venez 2007; 45: 74-8.

14. OMS. Encuestas de salud Bucodental. Métodos Básicos. Organización Mundial de la Salud. 4ta ed. Ginebra. 1997; 46-52.

15. Balzarini M.G., Gonzalez L., Tablada M., Casanoves F., Di Rienzo J.A., Robledo C.W. Infostat. Manual del Usuario. Ed. Brujas. Argentina. 2008.

16. Ayala Pérez Y; Carmona Vidal E. Maloclusiones y características clínicas de trastornos temporomandibulares en pacientes de la Clínica Manuel Angulo. Correo Científico Médico de Holguín 2010; 14 (2).

17. Folaranmi $\mathrm{N}$; Okeke A. A retrospective evaluation of the class of malocclusion amongst orthodontic patients at the University Of Nigeria Teaching Hospital (UNTH), Enugu, Nigeria. Ann Med Health Sci Res 20I I; (I): I03-6.

18. Gelgör IE, Karaman AI, Ercan E. Prevalence of malocclusion among adolescents in central anatolia. Eur J Dent. 2007; I (3): |25-3|.

19. Martins Mda G, Lima KC. Prevalence of malocclusions in 10to 12-year-old schoolchildren in Ceará, Brazil. Oral Health Prev Dent. 2009; 7 (3): 217-23.

20. Onyeaso CO. Prevalence of malocclusion among adolescents in Ibadan, Nigeria. Am J Orthod Dentofac Orthop. 2004;126:604-7.

21. Montiel ME. Frecuencia de maloclusiones y su asociación con hábitos perniciosos en una población de niños mexicanos de 6 a 12 años de edad. ADM. 2004;61:209-14.

22. Pantaleão dos Santos MR; Isper Garbin Aj; Saliba Garbin GA; Artênio PE; Garbin Jl. Estudio de las Características de Oclusión de Niños de 12 Años del Municipio de Cáceres, Mato Grosso, Brasil. Acta Odontológica Venez. 20I0; 48 (4): I-I0.

23. Roa Gonzales SC. Prevalencia de maloclusión en pacientes de siete $a$ doce años de edad: Un estudio epidemiológico. Med Oral;2008; 10: 16-20. 